\title{
Estimation of 2D Shear Wave Velocity Profile of Soil Layers Using Surface Wave Seismic Tests
}

\author{
Pei-hsun Tsai ${ }^{1}$ and Zheng-yi Feng ${ }^{2}$ \\ ${ }^{1}$ Department of Construction Engineering, Chaoyang University of Technology, Taiwan, R.O.C. \\ ${ }^{2}$ Department of Soil and Water Conservation, National Chung Hsing University, Taiwan, R.O.C.
}

\begin{abstract}
The 2D shear wave velocity profile of strata is estimated using the active and passive surface wave seismic tests. The experimental dispersion curves were obtained after the recorded signals were transformed by the slant stack procedure. The phase velocity in the relatively high frequency range can be obtained using the dispersion curves deduced from the active tests. On the other side, dispersion curves obtained from the passive tests can be used to estimate the phase velocity in the relatively low frequency range. From the higher frequency portion of the dispersion curves that stand for the fundamental mode, we obtained the phase velocities about $190 \mathrm{~m} / \mathrm{s}$ for the sandy surface fill. Theoretical dispersion curves can be constructed by the thin-layer-stiffness-matrix method. For theoretical dispersion curves, the soil layers of the test site were modeled as the sandy surface fill overlying a half space soil layer. A real-parameter genetic algorithm was programmed to minimize the difference between the theoretical and experimental dispersion curves. We prove that the real-parameter genetic algorithm is capable to reduce the error between experimental and theoretical dispersion curves. The estimated 2D geometry of the sandy surface fill using the active and passive surface wave seismic tests was verified with the borehole data.
\end{abstract}

\section{INTRODUCTION}

Shear wave velocity profile is an important part of seismic risk characterization. In order to analyze the safety of the buildings in Chaoyang University of Technology, Taiwan under earthquake loading, the shear wave velocity profile of soil layers need to be studied. Among the various seismic methods, surface wave seismic method is deemed as one of the best solutions. The Rayleigh waves travel on ground surface and they are easily detected using some receivers placed on ground surface. They contain plentiful dynamic information and they are useful for identification the soil strata in situ. Recently the development is the multichannel analysis of surface wave (MASW) method based on multiple channel records to construct the dispersion curve of Rayleigh wave. There are some analysis methods to evaluate the dispersion curve ${ }^{1-5)}$. The dispersion curve is selected from the MASW spectra.

\section{STUDY METHODS}

\section{(1) Surface wave seismic tests}

The study area is situated on Campus of the Chaoyang University of Technology, Taichung, Taiwan. The test setup used is shown in Fig. 1.
From the result of drilled borehole, the thickness of a fill is $5.7 \mathrm{~m}$ overlaid on dark gray weathered sandstone. The borehole (BH-1) is near to the study area (Fig. 1). The fill is a layer of gray sandy silt with little gravel, and it can be classified as sandy silt (SM) by the United Soils Classification System. The engineering properties of the fill from experimental work are as follows: unit weight $\gamma_{t}=$ $19.5 \mathrm{kN} / \mathrm{m}^{3}$, water content $\omega_{\mathrm{n}}=16 \%$, and friction angle $\phi=25^{\circ}$. The surface wave experimental data were collected using multiple receivers along a survey line. A surface wave seismic system consists of a 16-channels seismograph with 12 geophones of $4.5 \mathrm{~Hz}$ capacity mounted on $10 \mathrm{~cm}$ spikes had been used in this study. The 16-channel seismograph recorded the seismic data of each receiver. A linear array of 12 receivers $1 \mathrm{~m}$ apart aligned acquired the vertical velocity time history on ground surface. Receivers were aligned and spaced at $1 \mathrm{~m}$ interval. The spread length of the receivers was $11 \mathrm{~m}$. The spread of receivers were moved $1 \mathrm{~m}$ to northeast together along the survey line until the entire measurement process finished.

In active MASW test, the impulsive source was used by a sledgehammer of $10 \mathrm{~kg}$ impacting a $0.2 \mathrm{~m}$ by $0.2 \mathrm{~m}$ metal plate with a nearest source-toreceiver offset of $5 \mathrm{~m}$. The recording time was only 
$1 \mathrm{~s}$ with sampling rate of 5000 samples per second. The vibration source of passive MASW test was ambient noise. Because the study area is located in playground, some microtremor induced by persons running or playing ball. The duration of the seismic records was set to $40 \mathrm{~s}$ with sampling rate of 125 samples per second.

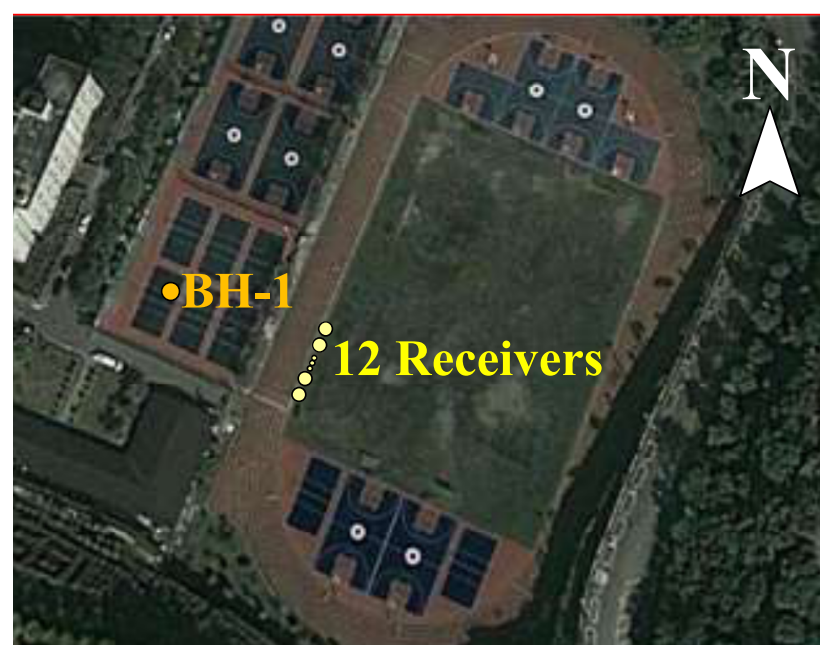

(a)

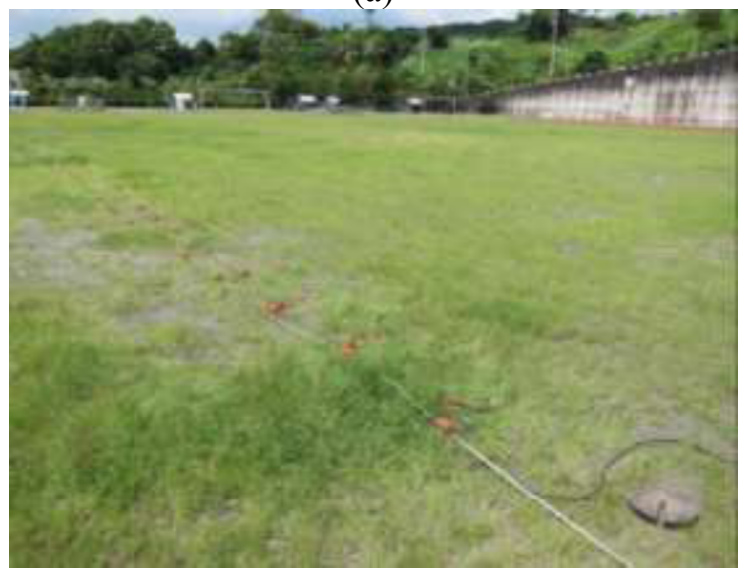

(b)

Figure 1 MASW method field configuration.

To obtain the shear wave velocity profile based on the experimental dispersion curves, the process involves three steps: measuring the vibration data along a survey line on ground surface, construction of dispersion curve, and back-calculation of the shear wave velocity profile from the measured dispersion curve.

\section{(2) Analysis of experimental dispersion curve}

Frequency vs. wave number (f-k) analysis and refraction microtremor (ReMi) method are two of the most recently developed MASW test for determining the shallow shear wave velocity profile.

The experimental dispersion curves for active MASW tests were obtained by Ryden et al. ${ }^{1)}$ proposed method. The peak of amplitude in an active MASW spectrum is assumed on the dispersion curve.

The ReMi method uses recording ambient noise or microtremor ubiquitous in the urban environment. The vibration data consisted of ambient noise records of 10 seconds duration in this study. They were transformed to the slowness-frequency (p-f) domain and stacked prior to dispersion analysis, as described by Louie ${ }^{4)}$. The experimental dispersion curve can be extracted from the lower limit of higher amplitude in the passive MASW spectra.

\section{ANALYSIS OF DISPERSION CURVE}

\section{(1) Active MASW test}

The frequency vs. phase velocity spectra at 10 seismic stations, close to the borehole, BH-1, in the study area by active MASW test are shown in Fig. 2. The seismic station number is sequentially assigned from south to north. The experimental fundamental mode dispersion curve can be selected from the peak values of the frequency-phase velocity spectrum. The evaluated dispersion curves are represented by a dashed white line with circle symbols and they are shown in the figures. Besides fundamental mode, there are numerous higher modes, as shown in Fig. 2. It can be seen from Fig. 2 that the 10 different spreads result in various dispersion curves especially in low frequencies. However, the asymptotes at high frequencies (greater than $40 \mathrm{~Hz}$ ) of the fundamental mode approach the phase velocities for the fill (sandy silt) of $190 \mathrm{~m} / \mathrm{s}$. Because the vibration induced by impulse loading in active MASW test, the duration of seismic records is less than that of passive MASW test. Therefore, it will be used to evaluate the feature of surface wave from medium to high frequency range due to its shorter duration. Because of the variations of dispersion curve in low frequencies, they will be further evaluated by passive MASW tests.

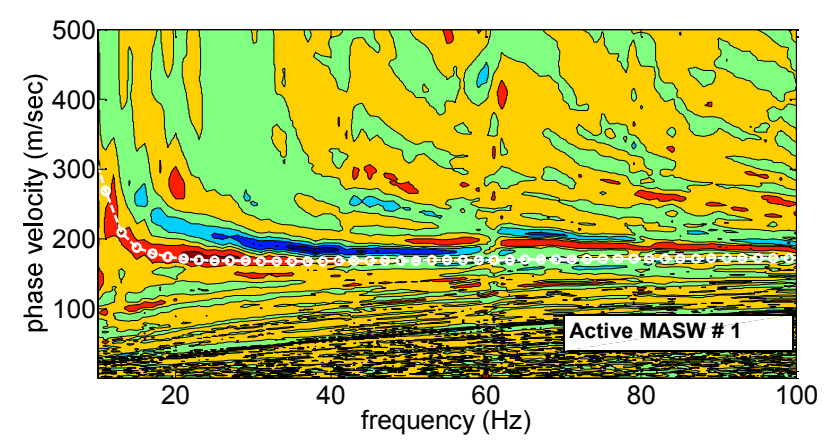



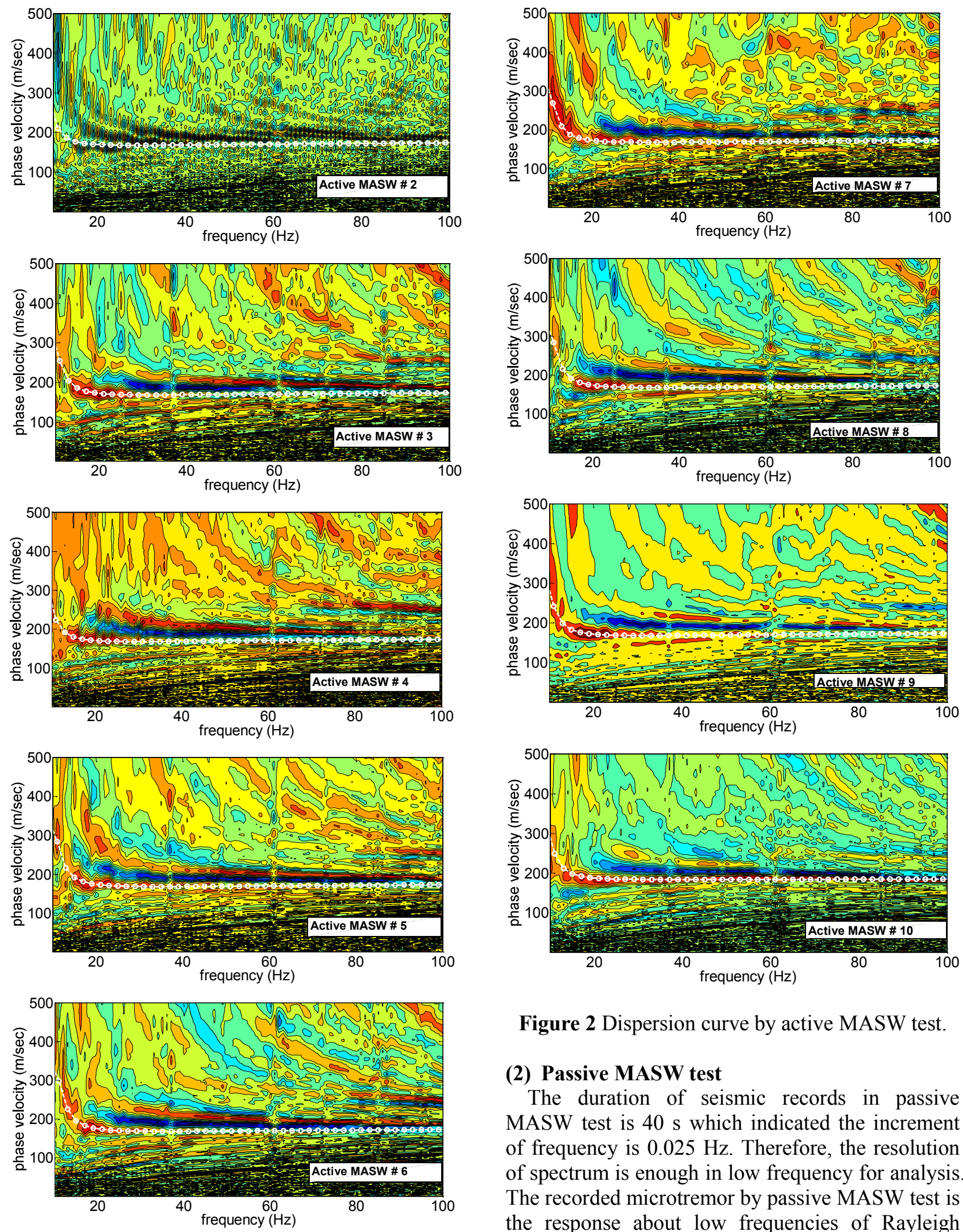

Figure 2 Dispersion curve by active MASW test.

\section{(2) Passive MASW test}

The duration of seismic records in passive MASW test is $40 \mathrm{~s}$ which indicated the increment of frequency is $0.025 \mathrm{~Hz}$. Therefore, the resolution of spectrum is enough in low frequency for analysis. The recorded microtremor by passive MASW test is the response about low frequencies of Rayleigh waves in the study area. It indicates the feature of surface wave in deep soil layers. The spectra at the 10 seismic stations in the study area by passive MASW test are shown in Fig. 3. The evaluated dispersion curves are represented by a dashed black line with circle symbols and they are shown in the 
figures.
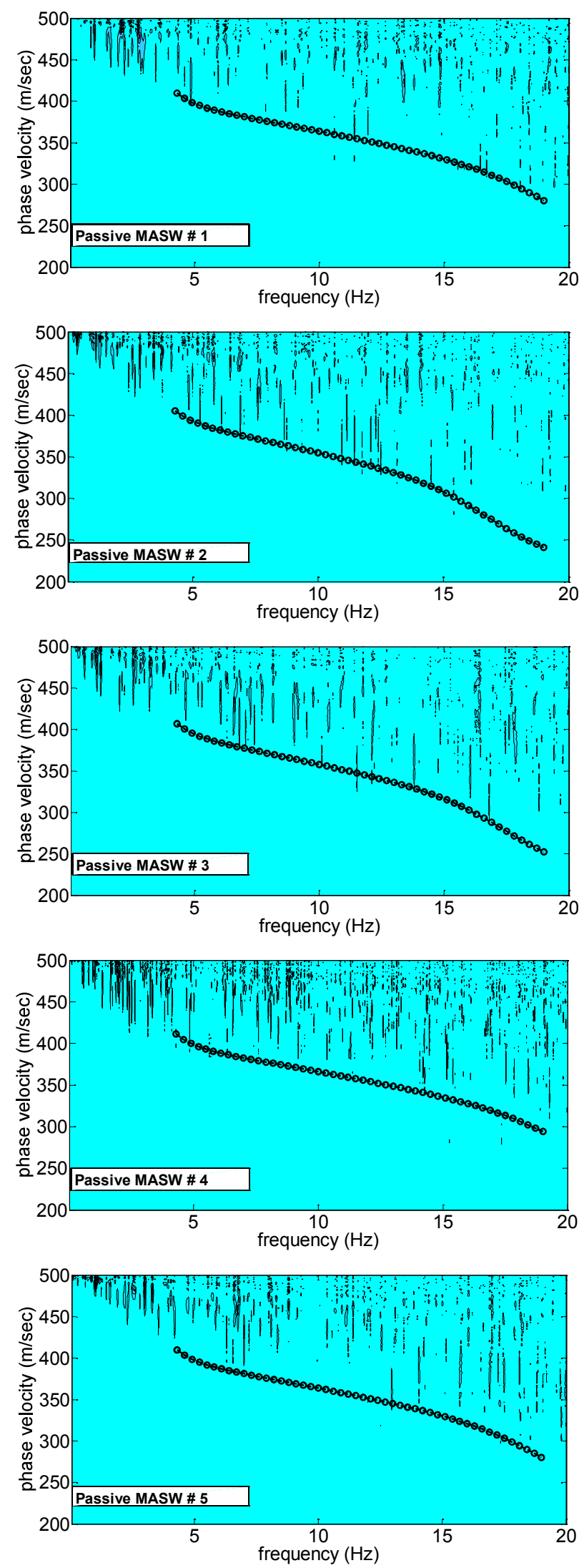
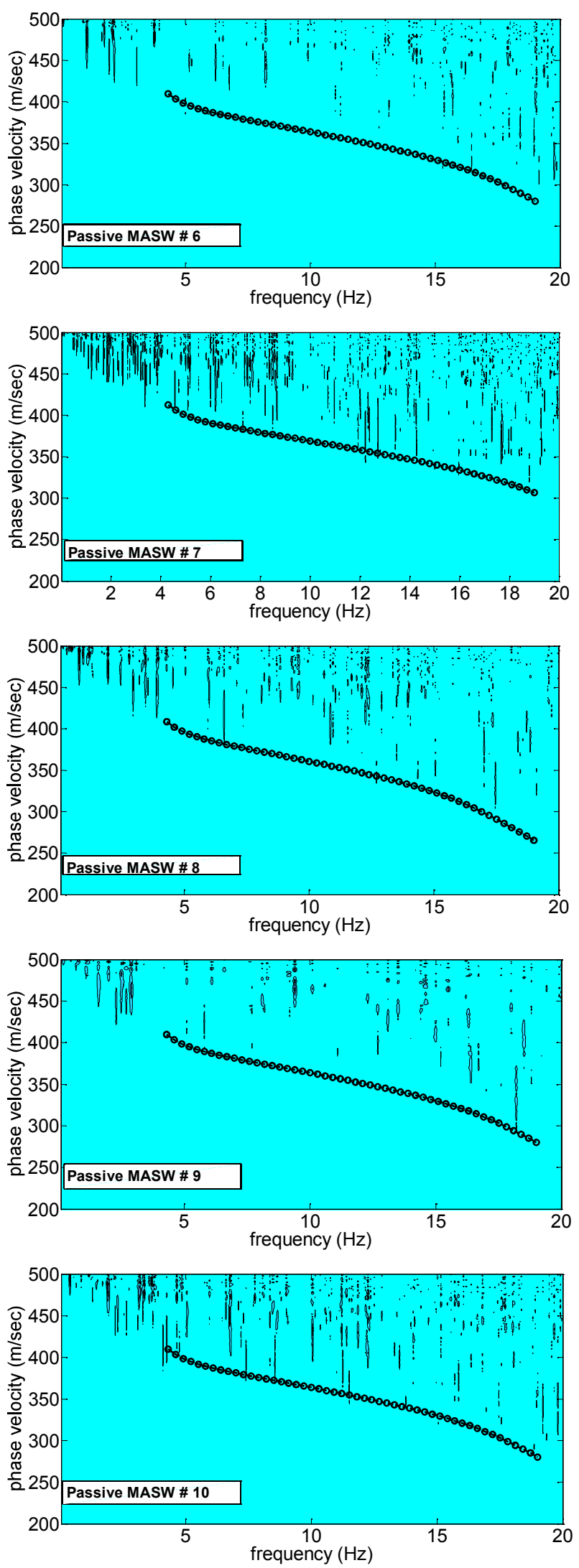

Figure 3 Dispersion curve by passive MASW test.

In Fig. 3, the dispersion curves at the 10 seismic stations are slightly different. This implies the shear wave velocity profiles of soil layers are similar. It 
can be because that the spacing between receivers is only $1 \mathrm{~m}$ and the length of survey line is only $10 \mathrm{~m}$.

\section{(3) Combined dispersion curves for active and passive MASW tests}

Combined active and passive seismic data can assist in reasonable characterization of soil layers. The dispersion curves for active and passive MASW test at the seismic station number of 1,4 and 7 are shown in Fig. 4. It can be seen from Fig. 4 that the dispersion curves for active and passive MASW tests are close in high frequencies. In low frequencies (less than $40 \mathrm{~Hz}$ ), the phase velocities for passive MASW tests are greater than that of active MASW test. The maximum difference of the both tests occurs in $18 \mathrm{~Hz}$. However, the difference is small when the frequency approaches to 8 or 40 $\mathrm{Hz}$.

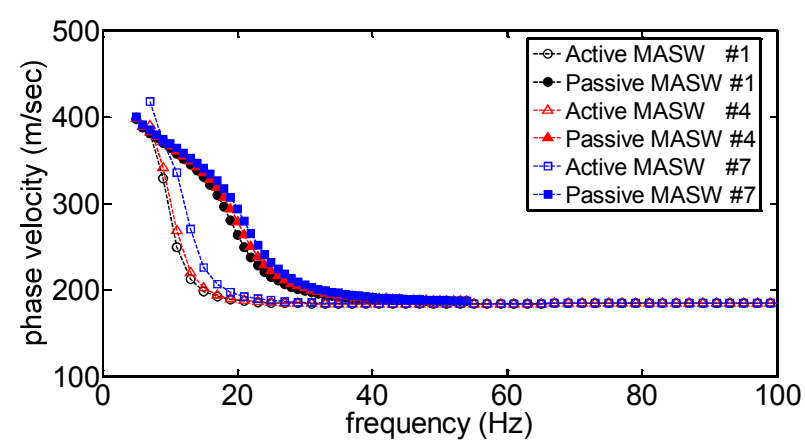

Figure 4 Comparison for dispersion curve of active and passive MASW test.

\section{SHEAR WAVE VELOCITY PROFILE}

We performed back-calculations to obtain shear wave velocity profiles based on the experimental dispersion curves. Theoretical dispersion curve can be constructed by thin layer stiffness matrix method $^{6}$. The soil layers are divided into some "thin" layers, firstly. Thin layers means that the layer thickness is smaller than the wavelength of interest. Assembling these layer matrices individually the stiffness matrix of the entire strata is setup. The stiffness matrix can be expressed as a function of wave number. Hence the eigenvalue problem for wave propagation is algebraic. And then, for the each frequency, the corresponding eigenvalue and eigenvector are obtained from these algebraic; they are wavenumber and modal shape for the individual frequency, respectively. The theoretical dispersion curve can be obtained with the relation of frequency and phase velocity of surface wave.

Genetic algorithm is a technique that searches an approximate solution for an optimization problem.
Such a solution is achieved based on an evolving process of numerical calculation. The solutions are selected through a fitness-based process in this study. The fitness of 50 sets of geo-material parameters is evaluated in each generation. There are four geo-material parameters of thin layer stiffness matrix method in a soil layer, such as layer thickness, shear wave velocity, Poisson's ratio and density. To save time of calculation, the densities of soils and weathered rock are fixed as 1800 and $2100 \mathrm{~kg} / \mathrm{m}^{3}$, respectively. The process is terminated after ten generations because the maximum of the fitness function has reached a steady value. The error of experimental curve and theoretical curve is within $5 \%$.

The recorded seismic data for active MASW test can indicate the shear wave velocity of soil near to ground surface. The depth of weathered bedrock is evaluated range from 6.4 to $8 \mathrm{~m}$ by using the dispersion curves from active MASW test. The variation of the depth of weathered bedrock is large. It is not reasonable because the length of survey line is only $10 \mathrm{~m}$. Additionally, they are different from the depth of bedrock obtained from the borehole test $(5.7 \mathrm{~m})$.

There is a high resolution of dispersion curve for passive MASW test in low frequencies. The depth of weathered bedrock is evaluated ranging from 4 to $4.8 \mathrm{~m}$. The results of the bedrock depth are more reliable than that from active tests. Therefore, we use the dispersion curves from passive MASW tests to evaluate the shear wave velocity and depth of weathered bedrock.

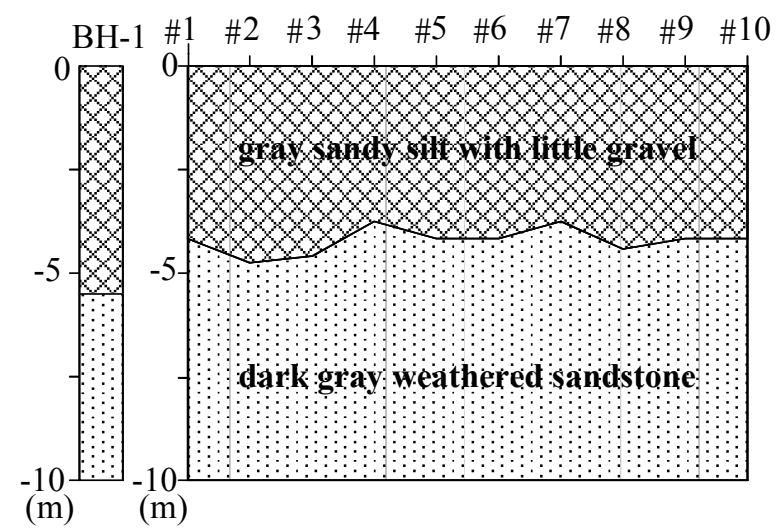

Figure 5 2D shear wave velocity profile.

The 2D shear wave velocity profile and borehole (BH-1) data are plotted in Fig. 5. The depths of weathered bedrock estimating from dispersion curves by passive MASW tests are from 4 to $4.8 \mathrm{~m}$ and the estimated depth is consistent with the borehole data. We conclude that for the test site, a sandy silt layer overlain on an underlying half space 


\section{CONCLUSIONS}

Based on the results of the MASW tests in this study, the following conclusions are made:

(1) The asymptotes at high frequencies of the fundamental mode dispersion curve of both active and passive MASW tests are close.

(2) The dispersion curve for passive MASW test in low frequencies has a high resolution.

(3) The estimated depth of weathered bedrock is consistent with the neighboring borehole data.

(4) The shear wave velocity and depth of weathered bedrock are suggested to be evaluated by the dispersion curves from the passive MASW test for this site.

(5) It is feasible using the real-parameter genetic algorithm to reduce the error of experimental and theoretical dispersion curve.

(6) The performance of passive MASW test is better than that of active MASW test in the test site.

ACKNOWLEDGMENT: This study is supported by the research funding from the National Science Council of Taiwan (NSC 101-2221-E-324-030- ). Their support is gratefully appreciated.

\section{REFERENCES}

1) Ryden, N., Park, C.B., Ulriksen, P., and Miller, R.D., 2004, Multimodal approach to seismic pavement testing, Journal of Geotechnical and Geoenvironmental Engineering, 130, 636-645.

2) Lu, L., and Zhang, B., 2004, Analysis of dispersion curves of Rayleigh waves in the frequency-wavenumber domain, Can. Geotech., 41, 583-598.

3) Lin, C.P., Chang, C.C., and Chang, T.S., 2004, The use of MASW method in the assessment of soil liquefaction potential, Soil Dynamics and Earthquake Engineering, 24, 689-698.

4) Louie, J. N., 2001, Faster, better: shear-wave velocity to 100 meters depth from refraction microtremor arrays, Bull. Seism. Soc. Am., 91, 347-364.

5) Mahajan, A.K., Galiana-Merino, J.J., Lindholm, C., Arora, B.R., Mundepi, A.K., Rai, N., and Chauhan, N., 2011, Characterization of the sedimentary cover at the Himalayan foothills using active and passive seismic techniques, Journal of Applied Geophysics, 73, 196-206.

6) Chai, H.Y., Phoon, K.K., Wei, C.F., and Lu, Y.F., 2011, Analysis of effects of active sources on observed phase velocity based on the thin layer method, Journal of Applied Geophysics, 73, 